\title{
The novel elicitor AsES triggers a defense response against Botrytis cinerea in Arabidopsis thaliana
}

\author{
V. Hael-Conrad ${ }^{\mathrm{a}, \mathrm{b}}$, E. Abou-Mansour a , J.-C. Díaz-Ricci ${ }^{\mathrm{b}}$, J.-P. Métraux $^{\mathrm{a}}$, M. Serrano ${ }^{\mathrm{a}, \mathrm{c}, *}$ \\ a Department of Biology, University of Fribourg, Chemin du Musée 10, 1700 Fribourg, Switzerland \\ b Instituto Superior de Investigaciones Biológicas (INSIBIO), CONICET-UNT, e Instituto de Química Biológica "Dr. Bernabé Bloj", Facultad de Bioquímica, \\ Química y Farmacia, UNT. Chacabuco 461, T4000ILI San Miguel de Tucumán, Argentina \\ ${ }^{c}$ Centro de Ciencias Genómicas, Universidad Nacional Autónoma de México, Avenida Universidad 2001, 62210 Cuernavaca, Morelos, Mexico
}

\begin{abstract}
AsES (Acremonium strictum Elicitor and Subtilisin) is a novel extracellular elicitor protein produced by the avirulent isolate SS71 of the opportunist strawberry fungal pathogen A. strictum. Here we describe the activity of AsES in the plant-pathogen system Arabidopsis thaliana-Botrytis cinerea. We show that AsES renders $A$. thaliana plants resistant to the necrotrophic pathogen $B$. cinerea, both locally and systemically and the defense response observed is dose-dependent. Systemic, but not local resistance is dependent on the length of exposure to AsES. The germination of the spores in vitro was not inhibited by AsES, implying that protection to $B$. cinerea is due to the induction of the plant defenses. These results were further supported by the findings that AsES differentially affects mutants impaired in the response to salicylic acid, jasmonic acid and ethylene, suggesting that AsES triggers the defense response through these three signaling pathways.
\end{abstract}

\section{Introduction}

Plants continually struggle against pathogens to survive. The mechanisms evolved by plants to protect themselves from pathogens comprise constitutive barriers and induced defense responses. The cuticle, the cell wall and phytoanticipins are pre-formed physical and chemical barriers that limit access of microbes to the plant cells $[1,2]$. Numerous pathogens can circumvent the constitutive defenses. However, plants can also recognize their aggressors and trigger defense mechanisms known as innate immune responses. Plant innate immunity consists in a suite of reactions induced at two levels. Firstly, plant trans-membrane pattern-recognition receptors (PRRs) recognize microbe-, pathogen- and/or damage-associated molecular patterns

Abbreviations: AsES, Acremonium strictum Elicitor and Subtilisin; hpti, hours prior to infection; hpi, hours post-inoculation; SA, salicylic acid; JA, jasmonic acid; ET, ethylene.

* Corresponding author at: Centro de Ciencias Genómicas, UNAM Avenida Universidad 2001, Col. Chamilpa, 62210 Cuernavaca, Morelos, Mexico. Fax: +52 7773175581 .

E-mail addresses: v.hael.conrad@hotmail.com (V. Hael-Conrad) eliane.abou-mansour@unifr.ch (E. Abou-Mansour),juan@fbqf.unt.edu.ar (J.-C. Díaz-Ricci), jean-pierre.metraux@unifr.ch (J.-P. Métraux), serrano@ccg.unam.mx (M. Serrano).
(MAMPs, PAMPs and/or DAMPs). This is referred to as PAMPtriggered immunity (PTI) $[3,4]$. Typical responses of PTI include the generation of reactive oxygen species (ROS), calcium $\left(\mathrm{Ca}^{2+}\right)$ influx from extracellular spaces and changes in free cytosolic $\mathrm{Ca}^{2+}$ concentrations, cell-wall alterations, production of phytoalexins, protein phosphorylation, activation of mitogen-activated protein kinase (MAPK) signaling pathways and induction of gene transcription providing protection against non-host pathogens and limiting disease [5-7]. If pathogens overcome PTI, the second level of defense takes place mainly inside the cell via the action of the specific resistance $(R)$ proteins characterized by conspicuous nucleotide-binding leucine-rich-repeats domains. $\mathrm{R}$ proteins recognize pathogen-delivered proteins called effectors. Successful microbes can secrete effectors that inhibit the plant defense mechanisms and promote the so-called effector-triggered susceptibility (ETS) [3]. However, if specific R proteins recognize the effectors, the effector-triggered immunity (ETI) is induced to limit the pathogen growth by a local programmed cell death or hypersensitive response (HR) $[5,8]$. In addition to the innate immune response that remains localized at the cellular site of pathogen invasion, recognition of pathogens by plants can also activate systemic acquired resistance (SAR), induced systemic resistance (ISR) and wound-induced resistance (WIR), that are well-studied to represent systemic defense responses. SAR, ISR and WIR play a key role in the signaling networks induced by PTI and ETI and involve the 
salicylic acid- (SA), jasmonic acid- (JA) and ethylene- (ET) induced pathways $[9,10]$. SA participates in SAR which is often characterized by localized necrosis and expression of pathogenesis related genes (PR) and, in general, is triggered by hemibiotrophic pathogens [11]. On the contrary, ISR is not associated with necrosis and is often triggered by necrotrophic pathogens [12] or upon colonization of roots by selected strains of nonpathogenic rhizobacteria [13]. Whereas WIR is typically elicited upon tissue damage such as that caused by insect feeding $[14,15]$. Defenses against necrotrophic pathogens and herbivorous insects are mostly regulated by JA/ET-dependent pathways $[14,16,17]$.

MAMPs such as polypeptides, glycoproteins, lipids, glycolipids, and oligosaccharides, as well as DAMPs released by microbial hydrolytic enzymes from plant components, such as cell-wall fragments, have been characterized and are known to trigger defense responses that require the activation of SA-, JA-, and ET-induced signaling pathways [18-20]. The novel elicitor AsES (Patent EPC $\mathrm{N}^{\circ} 12.720 .221 .6-1410$ ) is a member of subtilisinrelated alkaline proteases and its proteolytic activity is necessary to induce systemic defense responses in strawberry plants against Colletotrichum acutatum, a hemibiotrophic pathogen that is the causal agent of anthracnose disease in strawberry [21]. Upregulation of the SA-responsive defense genes PR1 and chitinase Chi2.1 was also observed, suggesting that the SA-signaling pathway is activated during AsES-triggered defenses. However, other details of the signaling pathways involved in AsES-triggered resistance are unknown.

Preliminary evidence showed that AsES also protects against the agriculturally important grey mold fungus Botrytis cinerea [22] but details on its action against this necrotrophic pathogen are missing [21]. In order to better understand the mode of action of AsES, we characterized its activity in the plant-pathogen system Arabidopsis thaliana-B. cinerea. Here we show that AsES triggers a defense response in dose- and time-dependent manner to $B$. cinerea, and SA-, JA- and ET-induced signaling pathways are fundamental to activate AsES-dependent responses.

The use of elicitors in agriculture to induce plant defenses could be an alternative to fungicides. However, a better understanding of the mode of action of elicitors is needed in order to optimize their activity and develop realistic applications for farmers [3].

\section{Material and methods}

\subsection{AsES purification and suspension}

AsES elicitor protein was purified as previously described [21]. Once purified, it was lyophilized and kept at $4{ }^{\circ} \mathrm{C}$ until use. It was re-suspended at the indicated concentration with distilled sterile water.

\subsection{Plant maintenance}

A. thaliana seeds were grown on a pasteurized soil mix of humus and perlite (3:1), kept at $4{ }^{\circ} \mathrm{C}$ for two days and then transferred to the growth chamber. Plants were grown during 4 weeks in a $12 \mathrm{~h}$ light $/ 2 \mathrm{~h}$ dark cycle with $60-70 \%$ of relative humidity, with a day temperature of $20-22{ }^{\circ} \mathrm{C}$ and a night temperature of $16-18^{\circ} \mathrm{C}$. A. thaliana ecotype Columbia-0 (Col-0) wild-type plants were obtained from the Nottingham Arabidopsis Stock Centre (Nottingham, UK), transgenic seeds over-expressing the salicylate hydroxylase NahG gene were obtained from J. Ryals [23]. The following $A$. thaliana mutants (all in the Col-0 background) were used and previously described: jar1-1, coi1-16, etr1-1, ein2-1 [24], ics1 [25], npr1 [26], and dde2.1 [27].

\subsection{Culture of B. cinerea, inoculation and staining of hyphae}

B. cinerea strain BMM was provided by Brigitte Mauch-Mani (University of Neuchatel, Switzerland). Growth, preparation of spore suspension and infection procedure were performed as previously described [28]. In all the experiments, mock- and AsEStreated plants were kept in trays covered with a transparent plastic dome to maintain high humidity and incubated under the same plant growth conditions. Mock-treated and B. cinerea-infected plants were kept at a similar temperature but in darkness, for 24 , 48, 72 and 96 hpi as stated in the figure legends.

Fungal hyphae and dead plant cells were stained by boiling inoculated leaves for $5 \mathrm{~min}$ in a solution of ethanolic lactophenol trypan blue. Stained leaves were cleared in chloral hydrate $\left(2.5 \mathrm{~g} \mathrm{ml}^{-1}\right)$ at room temperature by gentle shaking until a colored solution was no longer released. Then leaves were imbibed in glycerol $20 \%$ for $1 \mathrm{~h}$ and observed using a Leica DMR microscope with bright-field settings.

\subsection{AsES treatment}

Plants were pre-treated with $6 \mu \mathrm{l}$ droplets of $60 \mathrm{nM}$ AsES or mock (distilled sterile water) for $48 \mathrm{hpti}$. After this time the remaining droplets were removed and $6 \mu \mathrm{l}$ droplets containing the spore suspension $\left(5 \times 10^{4}\right.$ spores $\left.\mathrm{ml}^{-1}\right)$ were applied at the same location. Infection symptoms were evaluated 48 hpi by measurement of lesion size ( $\mathrm{mm})$. For the dose-response assay, plants were pretreated with $3,30,60,150,300$ or $1500 \mathrm{nM}$ AsES 48 hpti and evaluated at $48 \mathrm{hpi}$. For the pre-treatment and systemic assay, plants were pre-treated with $60 \mathrm{nM}$ AsES at 24, 48, 72, 96 and 120 hpti and evaluated at $48 \mathrm{hpi}$.

\subsection{B. cinerea infection of strawberries fruits}

Each strawberry was surface-sterilized with $50 \%(\mathrm{v} / \mathrm{v})$ ethanol, sprayed with $600 \mu \mathrm{l}$ of $60 \mathrm{nM}$ AsES, kept in high humidity inside transparent hermetic boxes and finally placed in a growth chamber under the same temperature and light conditions as $A$. thaliana plants for 48 hpti. B. cinerea spores were applied by spraying each strawberry with $600 \mu \mathrm{l}\left(5 \times 10^{4}\right.$ spores $\left.\mathrm{ml}^{-1}\right)$ and infection symptoms were evaluated $96 \mathrm{hpi}$.

\subsection{In-vitro effect of AsES on B. cinerea spores germination}

The in-vitro growth assay with $B$. cinerea was performed as previously described [24]. Briefly, a spore suspension of $B$. cinerea at a final concentration of $5 \times 10^{4}$ spores $\mathrm{ml}^{-1}$ and AsES (3, 30, 60, 150 or $300 \mathrm{nM}$ ) were mixed to a final volume of $30 \mu \mathrm{l}$. $10 \mu \mathrm{l}$ droplets were deposited on a slide and kept in high humid conditions inside dark hermetic boxes, placed in a growth chamber under the same conditions as for plant infections. Pictures were taken at 3,6 and $24 \mathrm{~h}$ post incubation with a digital camera attached to a Leica DMR microscope with bright-field settings. Images of growing $B$. cinerea hyphae were analyzed using Image J version $1.44(\mathrm{NIH})$.

\subsection{Quantification of $S A$ and camalexin}

SA and camalexin were quantified as previously described [29] and [30] respectively. For each biological replicate, treated leaves from six plants (corresponding to about $200 \mathrm{mg}$ fresh weight) were harvested, pooled and immediately frozen at $-20^{\circ} \mathrm{C}$. The amount of SA and camalexin was expressed in $\mathrm{ng} \mathrm{mg}^{-1}$ fresh weight (FW) and corrected with ortho-anisic acid as internal standard. 


\subsection{RNA extraction and real time RT-PCR}

Leaves were harvested, pooled and immediately frozen in liquid nitrogen and kept at $-80^{\circ} \mathrm{C}$ until use. RNA was prepared using the Trizol reagent containing $38 \%$ saturated phenol, $0.8 \mathrm{M}$ guanidine thiocyanate, $0.4 \mathrm{M}$ ammonium thiocyanate, $0.1 \mathrm{M}$ sodium acetate and $5 \%$ glycerol. RNA $(1 \mu \mathrm{g})$ was then retro-transcribed into cDNA according to manufacturer's indications (Omniscript RT kit, Qiagen, www.qiagen.com). qRT-PCR was performed using Sensimix SYBR Green Kit (Bioline, www.bioline.com). Gene expression values were normalized using the gene At4g26410 previously described as a stable reference gene [31].

The primers used to analyze the expression of the indicated gene were: PR1 (AT2G14610) PR1-fw 5'-TTCTTCCCTCGAAAGCTCAA and PR1-rev 5'-AAGGCCCACCAGAGTGTATG; AOS (AT5G42650) AOS-fw 5'-GTGGATTCTCGGCGATAAAA and AOS-rev 5'-ATCCAAAGATCTCCCGATCC; PDF1.2 (AT5G44420) PDF-fw 5'-CCAAGTGGGACATGGTCAG and PDF-rev 5'-ACTTGTGTGCTGGGAAGAC; and PR4 (AT3G04720) PR4-fw 5'-GTACCACCGCGGACTACTGT and PR4-rev 5'TGGAGGAATAAGCACTCACG.

\subsection{Statistical analysis}

The statistical analysis was carried out using the software InfoStat version 2013 (http://www.infostat.com.ar). Differences between means were evaluated by Student's $T$-Test ( $P$ value $\leq$ 0.01 or 0.05 as stated in the figure legend). ANOVA analysis was performed to detect significant variances among treatments and followed by Duncan test at a 99\% confidence level.

\section{Results}

\subsection{AsES protects against $\mathrm{B}$. cinerea infection}

The protein AsES has been described to provide protection in strawberry plants against the hemibiotrophic pathogen Colletotrichum acutatum [21]. To test if the elicitor has an effect in strawberry fruits towards infection with $B$. cinerea, $60 \mathrm{nM}$ AsES was applied on the fruits 48 hpti. Interestingly, 4 days after the inoculation of the pathogen, AsES-treated fruits did not show symptoms compared to the mock-treated ones (Supplementary Fig. 1). We tested whether AsES had a similar protective effect in A. thaliana inoculated with $B$. cinerea in 4 -weeks-old plants pre-treated with $60 \mathrm{nM}$ AsES $48 \mathrm{hpti}$. Two days after the infection, a $45 \%$ of reduction in the lesion size compared to mock-treated leaves was observed (Fig. 1). These results indicated that AsES has a protective effect against B. cinerea in A. thaliana plants as well as in strawberries.

\subsection{AsES does not affect germination of $\mathrm{B}$. cinerea spores}

To determine if AsES directly affects the germination of $B$. cinerea spores, we analyzed the development of the fungus in vitro and in planta. B. cinerea spores were germinated in vitro in the presence of different concentrations of AsES (from 3 to $300 \mathrm{nM}$ ) and hyphal growth was analyzed at 3, 6 and 24 hpi. Observations made under the microscope showed that none of the concentrations of AsES used had a clear inhibitory effect on hyphal growth (Supplementary Fig. 2A). Nevertheless, a quantitative analysis showed a minor, but significant, inhibition of hyphal growth at $3 \mathrm{hpi}$ at all the AsES concentrations compared to mock-treated samples (Fig. 2). However, this inhibitory effect was no longer observed at 6 hpi for any of the analyzed AsES concentrations (Fig. 2). Furthermore, analyzing hyphal growth on the surface of $A$. thaliana leaves indicated that no inhibition was observed at 24, 48 and 72 hpi using $60 \mathrm{nM}$ of AsES (Supplementary Fig. 2B). Taken together, these data indicate

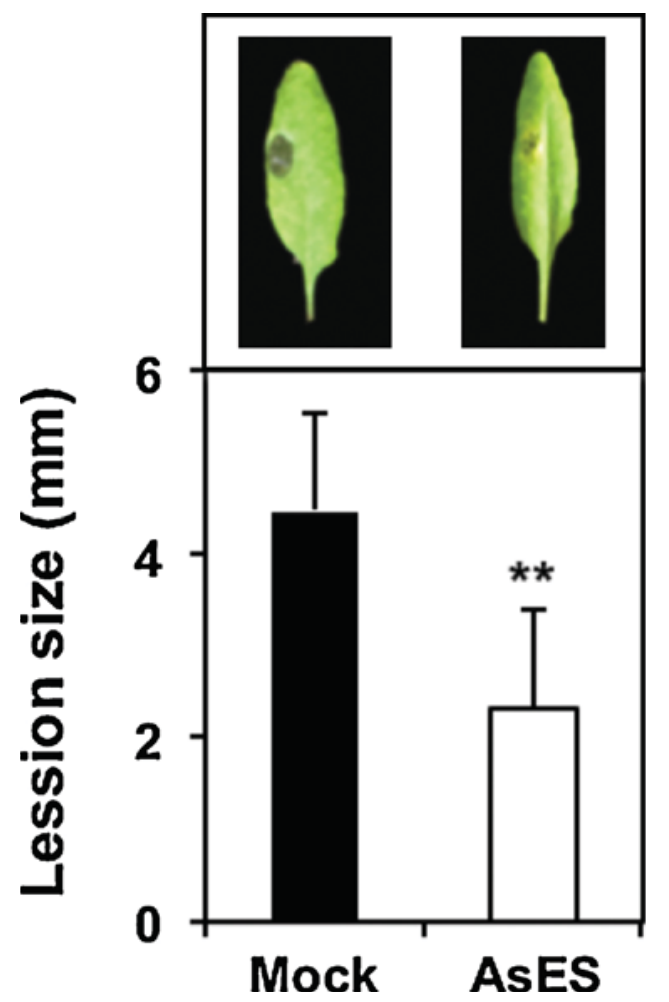

Fig. 1. Effect of AsES in A. thaliana plants infected with B. cinerea. Mock and AsES $(60 \mathrm{nM})$ treatments were applied $48 \mathrm{hpti}$ and lesion size was measured $48 \mathrm{hpi}$ with $B$. cinerea. Mean values \pm SE were obtained from five independent experiments $(n=20)$. A representative image of each treatment is presented. Asterisks indicate a statistically significant difference between mock- and AsES-treated plants, according to Student's $t$ test $(p<0.01)$.

that AsES does not affect the germination nor the initial development of the hyphae of $B$. cinerea, and the protective effect might be dependent on the modification of the plant defense responses.

\subsection{Dose-dependent effect of AsES against B. cinerea}

In order to evaluate the optimal concentration of AsES on the defense response of $A$. thaliana to $B$. cinerea, a dose-dependent curve was established. No protective effect compared to mocktreated control was observed using $3 \mathrm{nM}$ AsES on leaves infected with $B$. cinerea (Fig. 3A). However, at $30 \mathrm{nM}$, a $15 \%$ reduction in lesion size was observed with respect to control plants. At 60 and $150 \mathrm{nM}$ AsES a reduction of the lesion size of $43 \%$ and $55 \%$, respectively, was observed compared to control. Finally, when AsES was used either at 300 or $1500 \mathrm{nM}$ the reduction of lesion size was $66 \%$

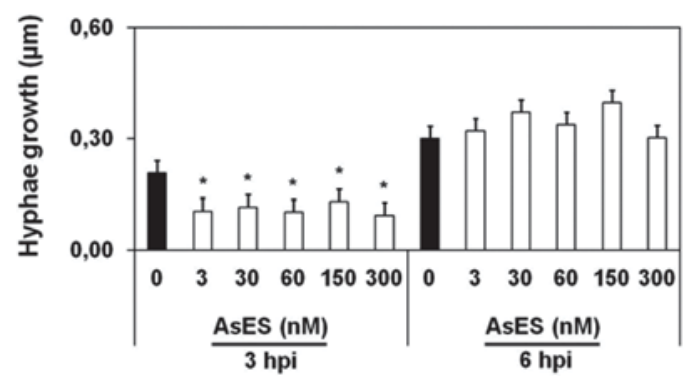

Fig. 2. Quantification of development of B. cinerea in the presence of AsES. Histogram of quantification of hyphae elongation at 3 and 6 hpi and different AsES concentrations. Mean values \pm SE were obtained from two independent experiments $(n=8)$. Asterisks indicate a statistically significant difference between the mock- and AsES-treated plants, according to Student's $t$ test $(p<0.01)$. 


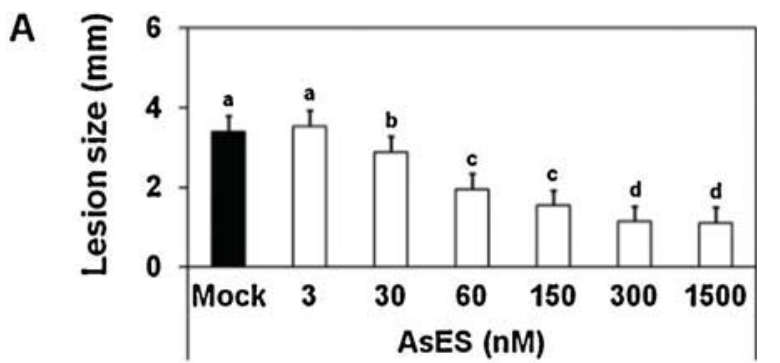

B

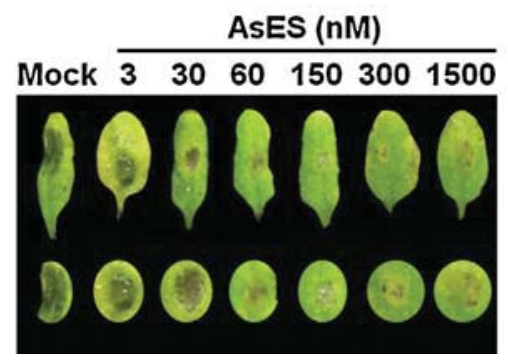

Fig. 3. Dose-dependent analysis of AsES in A. thaliana plants challenged with $B$. cinerea. (A) Lesions size of plants induced by different concentrations of AsES. (B) Appearance of Botrytis lesions on leaves pretreated with different concentrations of AsES; a close-up of the lesion is included in the lower panel. Mock and AsES treatments were applied 48 hpti and lesion size was measured 48 hpi. A representative image of each treatment is presented. Mean values \pm SE were obtained from three independent experiments $(n=20)$. Analysis of variance (ANOVA) followed by a Duncan test was performed using InfoStat/L software. Different letters represent statistically significant differences $(p<0.01)$.

with respect to mock-treated plants and was significantly different from that at 60 and $150 \mathrm{nM}$ (Fig. 3A). It is noteworthy that although protection was observed at $30 \mathrm{nM}$, the tissue was necrotized with water-soaking lesions that advanced through the leaf to the abaxial side (Fig. 3B). However, at higher concentrations the lesions were restricted to the adaxial side of leaves and they looked like drylesions, rising the hypothesis that it might be more difficult for the fungus to penetrate and infect the whole leaf (Fig. 3B).

\subsection{Time course of the effect of AsES against B. cinerea}

To further characterize the activity of AsES against B. cinerea, a time-course experiment was performed. Plants were treated with $60 \mathrm{nM}$ of AsES $48 \mathrm{hpti}$, and evaluated at 24, 48, 72 and $96 \mathrm{hpi}$. A significant effect with respect to mock-treated plants was observed at 48 and $72 \mathrm{hpi}$, with a reduction of symptoms (lesion size) of 45 and 20\%, respectively (Fig. 4). However, at 96 hpi no significant differences were observed compared to the controls, confirming a transitory effect of AsES during the first 72 hpi (Fig. 4). The macroscopic analysis of the infection caused by $B$. cinerea in planta in mock- and AsES-treated leaves, showed that at 24 hpi the symptoms were almost imperceptible for both treatments, while at 48 and 72 hpi the differences became significant (Supplementary Fig. 3). Interestingly, even though at 72 hpi the difference in the lesion size was less obvious between treated and control leaves, we observed that in AsES-treated leaves the lesion was generally less transparent and had a brown color rather than the black and water-soaked appearance of the mock-treated plants (closeup Supplementary Fig. 3) indicating to some extent, that they were more resistant. Moreover, even though the lesion size is statistically the same at $96 \mathrm{hpi}$, the morphology and color of both lesion types was different (close-up Supplementary Fig. 3). Since at 60 and $150 \mathrm{nM}$ there was no significant difference with respect to

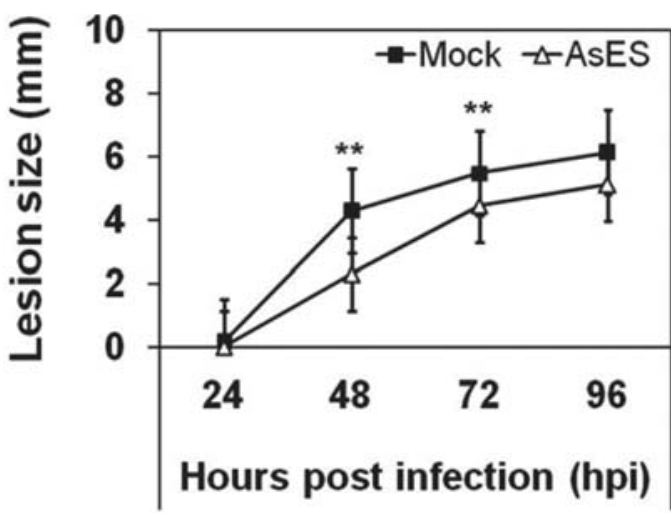

Fig. 4. Time-course of the development of lesion size caused by B. cinerea in AsEStreated A. thaliana plants. Mock and AsES $(60 \mathrm{nM})$ treatments were applied $48 \mathrm{hpti}$ and lesion size was measured $24,48,72$ and 96 hpi. Mean values \pm SE were obtained from three independent experiments $(n=20)$. Asterisks indicate a statistically significant difference between the mock- and AsES-treated plants, according to Student's $t$ test $(p<0.01)$.

lesion size, but only a change in the appearance of the lesions (Fig. 4 and Supplementary Fig. 3), we decided to carry out a time course with $150 \mathrm{nM}$ AsES (Supplementary Fig. 4A). Using $150 \mathrm{nM}$ AsES, the leaves were protected even at $96 \mathrm{hpi}$, compared to the protection using $60 \mathrm{nM}$ that was limited only up to $72 \mathrm{hpi}$. Moreover, lesions in AsES-treated leaves were much less transparent, had almost no brown color compared to the black and water-soaked appearance of the mock-treated plants, and infection was restricted to the initial droplet location (Supplementary Fig. 4B). This result indicates, once more, that increasing the concentration of AsES leads to a better and long-lasting inhibition of fungal growth.

\subsection{Effect of different timing in AsES pre-treatment against $\mathrm{B}$. cinerea}

To further evaluate the possibility of increasing the protection observed in plants treated 48 hpti with 60 nM AsES (45\% reduction of symptoms, Fig. 1), different pre-treatment times were assayed. Even at $24 \mathrm{hpti}$ the resistance against $B$. cinerea was triggered and the lesion size was reduced by $28 \%$ (Fig. 5). Additionally, at 72, 96 and $120 \mathrm{hpti}$, we determined that the lesion size was statistically the same as at 48 hpti. However, at longer times of pre-treatment the lesions were not sufficiently deep to reach the abaxial side of the leaves and looked like dry lesions compared to the water-soaked lesions observed at 48 hpti (Supplementary Fig. 5).

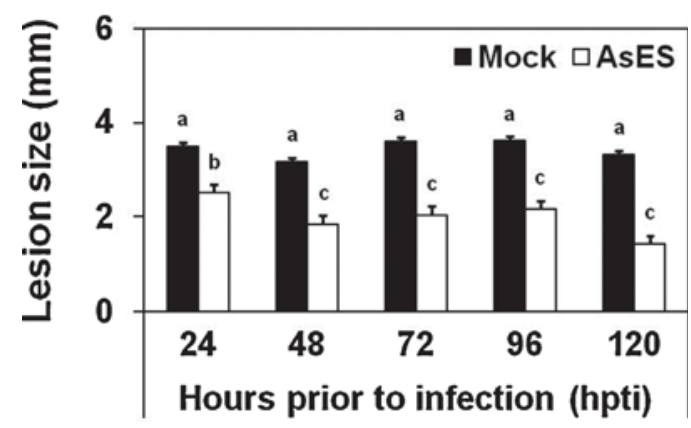

Fig. 5. Effect of different AsES pre-treatment times and B. cinerea infection in A. thaliana. Mock and AsES (60 nM) treatments were applied at different time points and lesion size was measured $48 \mathrm{hpi}$. Mean values \pm SE were obtained from three independent experiments $(n=20)$. Analysis of variance (ANOVA) followed by a Duncan test was performed using InfoStat/L software. Different letters represent statistically significant differences $(p<0.01)$. 
A

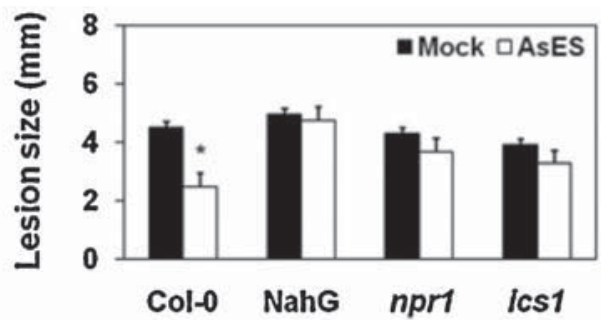

B

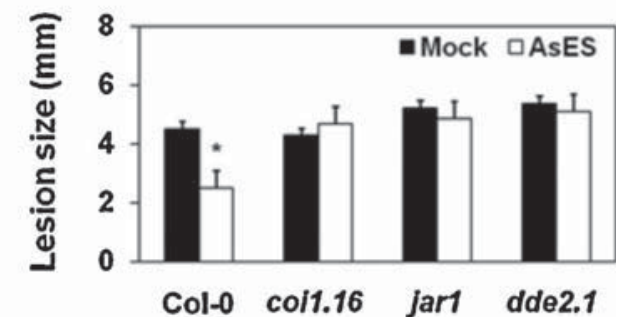

C

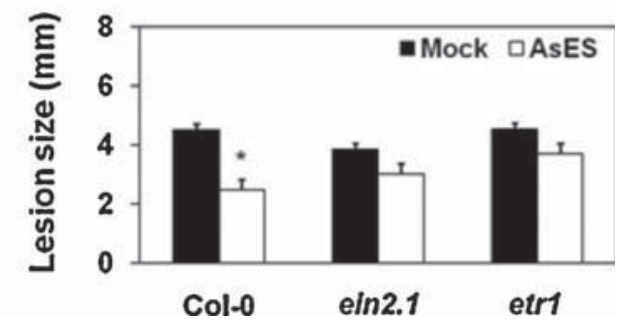

Fig. 6. Role of SA, JA, and ET in AsES-induced resistance to B. cinerea in A. thaliana. The transgenic plant NahG unable to accumulate $S A$, and the mutants npr1 and ics1 for the SA pathway (A), coi1.16, jar1, dde2.1 for JA (B), and ein2.1 and etr1 for ET (C) were evaluated. Mock and AsES (60 nM) treatments were applied $48 \mathrm{hpti}$ and lesion size was measured $48 \mathrm{hpi}$. Mean values \pm SE were obtained from four independent biological replicates $(n=20)$. Asterisks indicate a statistically significant difference between the respective mock- and AsES-treated plants, according to Student's $t$ test $(p<0.01)$.

3.6. Role of SA, JA, ET and camalexin in AsES-induced defense against $\mathrm{B}$. cinerea

Since AsES does not inhibit hyphal growth of $B$. cinerea (Fig. 2), it implies that AsES acts by an effect on plant defenses. To further confirm this hypothesis, we characterized the defense response induced by the AsES on mutants and transgenic plants affected in the major plant hormones SA, JA and ET. AsES-treated mutants impaired in the synthesis (ics1) and response (npr1), as well as transgenic plants impaired in SA accumulation (NahG) were all more susceptible to $B$. cinerea compared to mock-treated plants. Similarly, mutants affected in JA synthesis (dde2.1) and signaling (coi1.16 and jar1) or in ET perception (etr1) and signaling (ein2) were less resistant to $B$. cinerea after AsES treatment compared to controls (Fig. 6). These results indicate that SA, JA and ET are involved in the AsES-induced defense against $B$. cinerea infection.

Furthermore, a time course analysis of the expression of marker genes from the three signaling pathways PR1, PDF1.2 and PR4 and from the JA biosynthetic enzyme $A O S$, was performed. A previous report demonstrated that the expression of approximately onethird of the $A$. thaliana transcriptome changes during the first 48 hpi with $B$. cinerea [32]. Thus, we focused on this window. Total RNA was extracted from mock- and AsES-treated plants after 6, 24 and 48 hpti and at similar time points after $B$. cinerea infection and analyzed by qRT-PCR (Fig. 7). In AsES pre-treated leaves, a significant induction of PR1 was observed at 6 hpti, however the expression of PR1 decreased at $24 \mathrm{hpti}$ and remained stable at 48 hpti; in mock-treated plants PR1 expression was induced at $24 \mathrm{hpti}$ but decreased at $48 \mathrm{hpti}$ (Fig. 7A). A clear induction of PR1 expression was observed in infected plants at 6 hpi in AsES-treated leaves compared to mock-treated plants. At 24 and 48 hpi the expression in the mock- and AsES-treated leaves was similar albeit at a lower level (Fig. 7A). The expression of AOS in AsES-treated plants showed a significant inhibition at 6 hpti but no changes took place at 24 and 48 hpti. On the other hand, after $B$. cinerea infection, the expression of AOS increased 6 and 48 hpi in AsES-treated plants. The expression of the PDF1.2 gene was only slightly upregulated at $24 \mathrm{hpi}$ and to a lesser extent at $48 \mathrm{hpi}$ (Fig. 7B and C). Finally, the expression of $P R 4$ did not change in response to AsES, except at 6 hpti where the expression in AsES-treated leaves dropped significantly compared to untreated controls (Fig. 7D), although our experiments with mutants indicated a requirement for ET signaling.

To further analyze the SA-dependent defense response induced by AsES, free and conjugated SA were quantified in mock- or AsEStreated plants at $48 \mathrm{hpti}$ and after B. cinerea infection. At 24 and 48 hpi a slight increase in conjugated SA accumulation was observed (Supplementary Fig. 6).

To analyze if AsES-triggered defense responses were mediated by the accumulation of phytoalexin, camalexin was quantified in mock- and AsES-treated plants at 48 hpti and after B. cinerea infection. However, similar as SA quantification, no significant differences were observed (Supplementary Fig. 7).

A

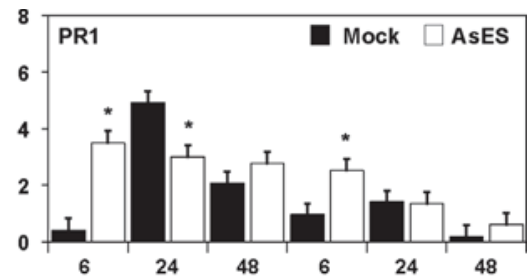

B

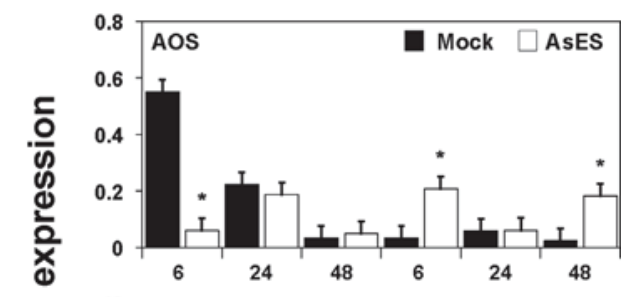

C

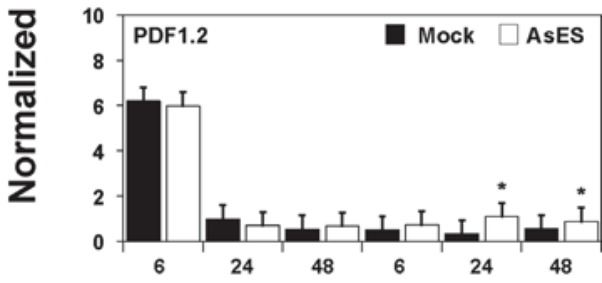

D

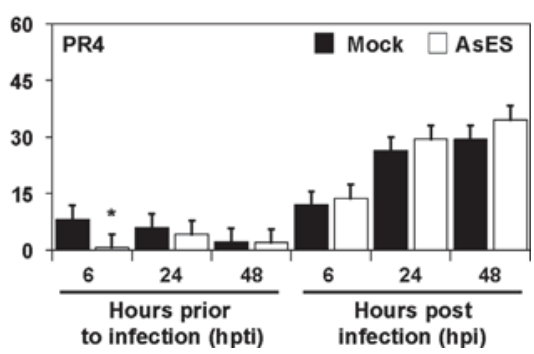

Fig. 7. Quantitative real-time PCR (qRT-PCR) analysis of marker genes of SA-,JA- and ET-induced defense pathways. The expression of selected genes was determined at 6, 24 and $48 \mathrm{hpti}$ and at 6,24 and $48 \mathrm{hpi}$ and normalized with respect to the reference gene QRT. Bars represent \pm SE from one experiment with three technical replicates for each qRT-PCR assay $(n=5)$. Asterisks indicate a statistically significant difference between the respective mock- and AsES-treated plants, according to Student's $t$ test $(p<0.01)$. 


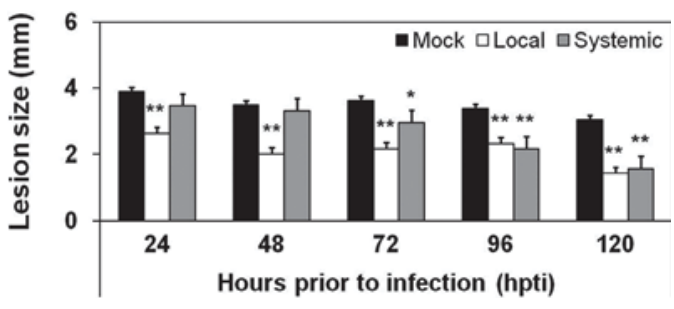

Fig. 8. Systemic effect triggered by AsES against $B$. cinerea in A. thaliana. Mock and AsES $(60 \mathrm{nM})$ treatments were applied at different time points and lesion size was measured 48 hpi. Mean values \pm SE were obtained from three independent experiments $(n=20)$. Asterisks indicate a statistically significant difference between the respective mock- and AsES-treated plants, according to Student's $t$ test $\left({ }^{*} p<0.05\right.$; $* * p<0.01$ ).

\subsection{AsES triggers a systemic resistance to $\mathrm{B}$. cinerea}

Since SA-related mutants were affected in AsES-induced resistance, and that AsES had systemic effect in strawberry plants $\{21\}$, we further assessed whether the elicitor triggered a systemic response in $A$. thaliana. At different times we pre-treated half of the leaves of the plant with $60 \mathrm{nM}$ of AsES (local) and the other half with $\mathrm{H}_{2} \mathrm{O}$ (systemic). After this pre-treatment we inoculated all the leaves with $B$. cinerea and measured the lesion size at 48 hpi. Leaves pre-treated for 24 and 48 hpti did not show a systemic protection, while the local leaves were protected (Fig. 8). However, leaves pre-treated with AsES for 72 hpti showed a slight defense response in the systemic leaves, while pre-treatment for 96 and 120 hpti showed a strong systemic defense response, similar to the response of local treated leaves (Fig. 8). This indicates that systemic induced resistance takes place only when the plant is induced by AsES for at least $72 \mathrm{hpti}$.

\section{Discussion}

4.1. AsES has the potential as a biocontrol agent against $\mathrm{B}$. cinerea

The necrotrophic fungus $B$. cinerea, commonly known as grey mold, has been classified as the second most important phytopathogen since it can infect over 200 plant species and destroy different organs of the plant during the pre- and post-harvest [22]. Elicitors represent an attractive potential alternative to fungicides since they can induce plant defense responses [3]. Numerous elicitors of different nature have been previously described to protect plants against $B$. cinerea, such as rhamnolipids (RLs) [33], oligogalacturonides (OGs) [33,34], chitosan [35] and its quaternized oligomers (QCOS) [35,36], a heat-stable protein PebC1 [37] and ceratoplatanin $(\mathrm{CP})$ [38]. In this work we characterized the protective effect of the novel elicitor AsES against $B$. cinerea. We show that prophylactic application of AsES on either A. thaliana leaves and Fragaria sp. fruits $48 \mathrm{hpti}$ leads to a significant inhibition of $B$. cinerea growth (Fig. 1 and Supplementary Fig. 1). Moreover, AsES does not cause inhibition of $B$. cinerea in vitro nor in planta at 24 and 48 hpi even at the highest dose tested (Fig. 2 and Supplementary Fig. $2 \mathrm{~A}$ and $\mathrm{B}$ ). This result suggests that the induced protection is most likely due to plant defenses rather than direct toxicity to the fungus. The dose-dependent effect of AsES (Fig. 3) is in agreement with observations made with other elicitors acting against $B$. cinerea on A. thaliana plants $[31,35]$. The length of the pretreatment period is not crucial for triggering resistance, but longer pretreatments lead to improved resistance (Fig. 5). Since AsES is not acting directly against $B$. cinerea (Fig. 2), the lag period is likely to be necessary for AsES to penetrate at the appropriate location and trigger defense responses in the plant. Together these results indicate that AsES has the potential as a biocontrol agent against $B$. cinerea.
4.2. AsES induces plant defenses against B. cinerea via SA-, JAand ET-signaling pathways

An effect of AsES via induced plant defenses is substantiated by our result with NahG plants or with mutants impaired in SA-, JA- and ET-signaling pathways (Fig. 6). Interestingly, the protective effect of AsES against B. cinerea depended on the SA-, JA- and ET-signaling pathways indicating that a diversity of defenses that depend on a combination of signaling pathways are activated by AsES. Other studies have reported that basic defenses of $A$. thaliana to $B$. cinerea depend mostly on JA and ET $[16,17,39]$. Nevertheless, SA-induced plant defenses were also reported to be involved against necrotrophs in A. thaliana [39-41] and tobacco [42]. Perhaps a difference with the studies cited above and our results is that we have tested the hormone dependence of elicitor-triggered defenses in contrast to hormone-dependence of basal resistance without elicitor pretreatment. To further confirm the participation of the three phytohormones we analyzed gene expression of SA-, JA-, and ET-responsive genes in AsES-induced plants. We observed upregulation of the SA- and JA-induced genes PR1 and PDF1.2, respectively, as well as the biosynthetic JA enzyme AOS (Fig. 7). However the ET-induced gene PR4 was not induced over its control value. Since we had demonstrated that ET-related mutants play a role in AsES-induced defense (Fig. 6C), one possible explanation to this result is that other ET-responsive genes might participate, but were not included in the analysis. Cross-talk between defensesignaling pathways has been well documented $[43,44]$ and is likely to contribute to optimize the defenses depending on the type of challenge. A crosstalk might possibly occur in AsES-induced defenses between SA- and JA-induced genes. For example PR1 is strongly expressed at $6 \mathrm{hpti}$ while PDF1.2 is not and AOS is even strongly repressed (Fig. 7). This reverses at 6, 24 and 48 hpi where PR1 is only induced at 6 hpi, while PDF1.2 at 24 and 48 hpi and AOS at 6 and 48 hpi are expressed (Fig. 7). Similar cross-talk events have been described for the $B$. cinerea-induced protein kinase mutant bik1, that shows inhibition of PDF1.2 but increased SA-induced defense responses [45] and for the activator of SA and repressor of JA-responsive genes, the transcription factor WRKY70 [46,47]. More work would be required to determine the exact localization of these cross-talks in relation to pathogen invasion to determine their relevance.

Our data on the quantification of conjugated SA indicate that the amount of conjugated SA had increased at 24 and 48 hpi in AsES-treated plants compared to the mock-treated controls (Supplementary Fig. 6). It is likely that AsES only needs basal levels of free SA for its action. A transient elevation in free SA that remained undetected might be another possibility; an argument in favor of this would be the elevation in conjugated SA detected at later timepoints. Since PR1 gene expression has mostly been associated with an increase in SA, this result is somewhat in contrast with the over-expression of PR1 at 6 hpti (Fig. 7). Similar to our findings, Méndez-Bravo et al. showed that $A$. thaliana plants pre-treated with $\mathrm{N}$-isobutyl decanamide, an alkamide present in plants, did not significantly affect the overall SA content, despite upregulation of PR1 [48]. These data suggests that alkamide-mediated gene expression of PR1 is independent of SA accumulation. Further experiments would be needed to clarify if PR1 is induced by AsES independently of SA.

\subsection{AsES also induces a systemic defense response against B. cinerea}

Finally, we assessed whether AsES has the potential to trigger a systemic response in A. thaliana, as previously shown in strawberry plants [21]. We observed a systemic effect of AsES in A. thaliana plants against $B$. cinerea. The effect is slightly detectable at $72 \mathrm{hpti}$, 
and stronger and as effective as the respective local treatments at 96 and 120 hpti (Fig. 8). Based on these results, we assume that AsES needs at least $24 \mathrm{~h}$ to trigger local acquired resistance and between 72 and $120 \mathrm{~h}$ to activate SAR which maintains the whole plant alert to possible future infections.

\section{Conclusion}

In conclusion, AsES exhibits local and systemic defense resistance on the non-host $A$. thaliana against the necrotrophic pathogen $B$. cinerea. AsES does not inhibit the fungal growth in vitro nor in planta, and requires SA-, JA- and ET-dependent signaling, supporting the notion that this elicitor stimulates defense responses in $A$. thaliana. Whether AsES or AsES-derived products act as MAPMs or DAMPs remains unknown. Future work should now be directed at determining the nature of the product recognized by the plant and the components involved in their recognition.

\section{Authors' contributions}

Conceived and designed the experiments: VHC, JCDR, JPM and MS. Performed the experiments: VHC. Performed SA and CA quantification: EA-M. Analyzed the data: VHC, JCDR, JPM and MS. Wrote the paper: VHC, JCDR, JPM and MS.

\section{Acknowledgements}

This work was made possible by funds to JPM from the Swiss National Science Foundation and the Argentinian grants PICT 20132075 and CIUNT 26-544. VHC is CONICET fellow and was supported by a fellowship granted by Programa de Becas de formación en el exterior en Ciencia y Tecnología (BEC.AR) and CONICET. JCDR is member of CONICET. The funding agencies had no role in study, design, collection, analysis and interpretation of data, decision to publish, or writing of the report. We thank Ms. Linda Grainger for her invaluable technical assistance. Thanks are also extended to Antony Buchala for critical reviewing of the manuscript.

\section{Appendix A. Supplementary data}

Supplementary data associated with this article can be found, in the online version, at http://dx.doi.org/10.1016/j.plantsci.2015.09. 025 .

\section{References}

[1] W. Underwood, The plant cell wall: a dynamic barrier against pathogen invasion, Front. Plant Sci. 3 (2012).

[2] T. Newman, J.T. Sundelin, MAMP (microbe-associated molecular pattern) triggered immunity in plants, Front. Plant Sci. 4 (2013).

[3] A.C. Wiesel, I. Newton, D. Elliott, E.M. Booty, P.R.J. Gilroy, Molecular effects of resistance elicitors from biological origin and their potential for crop protection, Front. Plant Sci. 5 (2014).

[4] C. Zipfel, Plant pattern-recognition receptors, Trends Immunol. 35 (2014) 345-351.

[5] J.D.G. Jones, J.L. Dangl, The plant immune system, Nature 444 (2006) 323-329.

[6] T. Boller, G. Felix, A renaissance of elicitors: perception of microbe-associated molecular patterns and danger signals by pattern-recognition receptors, Annu. Rev. Plant Biol. 60 (2009) 379-406.

[7] C. Garcion, O. Lamotte, J.P. Métraux, Mechanisms of defence to pathogens: biochemistry and physiology, in: W.D.A. Newton, G.D. Lyon (Eds.), Induced Resistance for Plant Defence: A Sustainable Approach to Crop Protection, Blackwell Press, Oxford, 2007, pp. 109-132.

[8] L. Deslandes, S. Rivas, Catch me if you can: bacterial effectors and plan targets, Trends Plant Sci. 17 (2012) 644-655.

[9] A. Robert-Seilaniantz, L. Navarro, R. Bari, J.D.G. Jones, Pathological hormone imbalances, Curr. Opin. Plant Biol. 10 (2007) 372-379.

[10] A. Robert-Seilaniantz, M. Grant, J.D.G. Jones, Hormone crosstalk in plant disease and defense: more than just jasmonate-salicylate antagonism, Annu. Rev. Phytopathol. 49 (2011) 317-343.
[11] A.C. Vlot, D.M.A. Dempsey, D.F. Klessig, Salicylic acid, a multifaceted hormone to combat disease, Annu. Rev. Phytopathol. 47 (2009) 177-206.

[12] B.P.H.J. Thomma, K. Eggermont, I.A.M.A. Penninckx, B. Mauch-Mani, R. Vogelsang, B.P.A. Cammue, W.F. Broekaert, Separate jasmonate-dependent and salicylate-dependent defense-response pathways in Arabidopsis are essential for resistance to distinct microbial pathogens, Proc. Natl. Acad. Sci. U. S. A. 95 (1998) 15107-15111.

[13] L.C. Van Loon, P.A.H.M. Bakker, C.M.J. Pieterse, Systemic resistance induced by rhizosphere bacteria, Annu. Rev. Phytopathol. 36 (1998) 453-483.

[14] A. Kessler, I.T. Baldwin, Plant responses to insect herbivory: the emerging molecular analysis, Annu. Rev. Plant Biol. 53 (2002) 299-328.

[15] G.A. Howe, Jasmonates as signals in the wound response, J. Plant Growth Regul. 23 (2004) 223-237.

[16] B.P.H.J. Thomma, I.A.M.A. Penninckx, W.F. Broekaert, B.P.A. Cammue, The complexity of disease signaling in Arabidopsis, Curr. Opin. Immunol. 13 (2001) 63-68.

[17] J. Glazebrook, Contrasting mechanisms of defense against biotrophic and necrotrophic pathogens, Annu. Rev. Phytopathol. 43 (2005) 205-227.

[18] F. Katagiri, K. Tsuda, Understanding the plant immune system, Mol. Plant Microbe. Interact. 23 (2010) 1531-1536.

[19] K. Tsuda, M. Sato, T. Stoddard, J. Glazebrook, F. Katagiri, Network properties of robust immunity in plants, PLoS Genet. 5 (2009) e1000772.

[20] M.E. Maffei, G.-I. Arimura, A. Mithofer, Natural elicitors, effectors and modulators of plant responses, Nat. Prod. Rep. 29 (2012) 1288-1303

[21] N.R. Chalfoun, C.F. Grellet-Bournonville, M.G. Martínez-Zamora, A.

Díaz-Perales, A.P. Castagnaro, J.C. Díaz-Ricci, Purification and characterization of AsES protein: a subtilisin secreted by Acremonium strictum is a novel plant defense elicitor, J. Biol. Chem. 288 (2013) 14098-14113.

[22] R. Dean, J.A.L. Van Kan, Z.A. Pretorius, K.E. Hammond-Kosack, A. Di Pietro, P.D. Spanu, J.J. Rudd, M. Dickman, R. Kahmann, J. Ellis, G.D. Foster, The top 10 fungal pathogens in molecular plant pathology, Mol. Plant Pathol. 13 (2012) 414-430.

[23] T. Gaffney, L. Friedrich, B. Vernooij, D. Negrotto, G. Nye, S. Uknes, E. Ward, H. Kessmann, J. Ryals, Requirement of salicylic acid for the induction of systemic acquired resistance, Science 261 (1993) 754-756.

[24] C. Chassot, C. Nawrath, J.P. Metraux, Cuticular defects lead to full immunity to a major plant pathogen, Plant J. 49 (2007) 972-980.

[25] M.C. Wildermuth, J. Dewdney, G. Wu, F.M. Ausubel, Isochorismate synthase is required to synthesize salicylic acid for plant defence, Nature 417 (2001) $562-565$.

[26] H. Cao, S.A. Bowling, A.S. Gordon, X. Dong, Characterization of an Arabidopsis mutant that is nonresponsive to inducers of systemic acquired resistance, Plant Cell 6 (1994) 1583-1592.

[27] P.M. Sanders, P.Y. Lee, C. Biesgen, J.D. Boone, T.P. Beals, E.W. Weiler, R.B. Goldberg, The Arabidopsis delayed Dehiscence1 gene encodes an enzyme in the jasmonic acid synthesis pathway, Plant Cell 12 (2000) 1041-1062.

[28] F. L'Haridon, A. Besson-Bard, M. Binda, M. Serrano, E. Abou-Mansour, F. Balet, H.-J. Schoonbeek, S. Hess, R. Mir, J. Léon, O. Lamotte, J.-P. Métraux, A permeable cuticle is associated with the release of reactive oxygen species and induction of innate immunity, PLoS Pathog. 7 (2011) e1002148.

[29] C. Fragnière, M. Serrano, E. Abou-Mansour, J.-P. Métraux, F. L'Haridon, Salicylic acid and its location in response to biotic and abiotic stress, FEBS Lett. 585 (2011) 1847-1852.

[30] C. Chassot, A. Buchala, H. Schoonbeek, J.P. Metraux, O. Lamotte, Wounding of Arabidopsis leaves causes a powerful but transient protection against Botrytis infection, Plant J. 55 (2008) 555-567.

[31] T. Czechowski, M. Stitt, T. Altmann, M.K. Udvardi, W.R. Scheible, Genome-wide identification and testing of superior reference genes for transcript normalization in Arabidopsis, Plant Physiol. 139 (2005) 5-17.

[32] O. Windram, P. Madhou, S. McHattie, C. Hill, R. Hickman, E. Cooke, D.J. Jenkins, C.A. Penfold, L. Baxter, E. Breeze, S.J. Kiddle, J. Rhodes, S. Atwell, D.J. Kliebenstein, Y. -s. Kim, O. Stegle, K. Borgwardt, C. Zhang, A. Tabrett, R. Legaie, J. Moore, B. Finkenstadt, D.L. Wild, A. Mead, D. Rand, J. Beynon, S. Ott, V. Buchanan-Wollaston, K.J. Denby, Arabidopsis defense against Botrytis cinerea: chronology and regulation deciphered by high-resolution temporal transcriptomic analysis, Plant Cell Online 24 (2012) 3530-3557.

[33] L. Sanchez, B. Courteaux, J. Hubert, S. Kauffmann, J.-H. Renault, C. Clément, F. Baillieul, S. Dorey, Rhamnolipids elicit defense responses and induce disease resistance against biotrophic, hemibiotrophic, and necrotrophic pathogens that require different signaling pathways in Arabidopsis and highlight a central role for salicylic acid, Plant Physiol. 160 (2012) 1630-1641.

[34] S. Ferrari, R. Galletti, C. Denoux, G. De Lorenzo, F.M. Ausubel, J. Dewdney, Resistance to Botrytis cinerea induced in Arabidopsis by elicitors is independent of salicylic acid, ethylene, or jasmonate signaling but requires PHYTOALEXIN DEFICIENT3, Plant Physiol. 144 (2007) 367-379.

[35] P. Trotel-Aziz, M. Couderchet, G. Vernet, A. Aziz, Chitosan stimulates defense reactions in grapevine leaves and inhibits development of Botrytis cinerea, Eur. J. Plant Pathol. 114 (2006) 405-413.

[36] H. Feng, W. Xia, C. Shan, T. Zhou, W. Cai, W. Zhang, Quaternized chitosan oligomers as novel elicitors inducing protection against $B$. cinerea in Arabidopsis, Int. J. Biol. Macromol. 72 (2015) 364-369.

[37] Y. Zhang, X. Yang, H. Zeng, L. Guo, J. Yuan, D. Qiu, Fungal elicitor protein PebC from Botrytis cinerea improves disease resistance in Arabidopsis thaliana, Biotechnol. Lett. 36 (2014) 1069-1078.

[38] L. Baccelli, S. Lombardi, R. Luti, P. Bernardi, A. Picciarelli, Cerato-platanin induces resistance in Arabidopsis leaves through stomatal perception, 
overexpression of salicylic acid- and ethylene-signalling genes and camalexin biosynthesis, PLoS One 9 (2014) e100959.

[39] S. Ferrari, J.M. Plotnikova, G. De Lorenzo, F.M. Ausubel, Arabidopsis local resistance to Botrytis cinerea involves salicylic acid and camalexin and requires EDS4 and PAD2, but not SID2, EDS5 or PAD4, Plant J. 35 (2003) 193-205.

[40] E.M. Govrin, A. Levine, Infection of Arabidopsis with a necrotrophic pathogen Botrytis cinerea, elicits various defense responses but does not induce systemic acquired resistance (SAR), Plant Mol. Biol. 48 (2002) 267-276.

[41] V.A. Halim, S. Altmann, D. Ellinger, L. Eschen-Lippold, O. Miersch, D. Scheel, S Rosahl, PAMP-induced defense responses in potato require both salicylic acid and jasmonic acid, Plant J. 57 (2009) 230-242.

[42] R. Menard, S. Alban, P. de Ruffray, F. Jamois, G. Franz, B. Fritig, J.-C. Yvin, S. Kauffmann, \{beta $\}-1,3$ glucan sulfate, but not $\{$ beta $\}-1,3$ glucan, induces the salicylic acid signaling pathway in tobacco and Arabidopsis, Plant Cell 16 2004) 3020-3032.

[43] A. Koornneef, C.M.J. Pieterse, Cross talk in defense signaling, Plant Physiol. 146 (2008) 839-844.

[44] C.M.J. Pieterse, A. Leon-Reyes, S. Van der Ent, S.C.M. Van Wees, Networking by small-molecule hormones in plant immunity, Nat. Chem. Biol. 5 (2009) $308-316$.
[45] P. Veronese, H. Nakagami, B. Bluhm, S. AbuQamar, X. Chen, J. Salmeron, R.A. Dietrich, H. Hirt, T. Mengiste, The membrane-anchored BOTRYTIS-INDUCED KINASE1 plays distinct roles in Arabidopsis resistance to necrotrophic and biotrophic pathogens, Plant Cell 18 (2006) 257-273.

[46] S. AbuQamar, X. Chen, R. Dhawan, B. Bluhm, J. Salmeron, S. Lam, R.A. Dietrich, T. Mengiste, Expression profiling and mutant analysis reveals complex regulatory networks involved in Arabidopsis response to Botrytis infection, Plant J. 48 (2006) 28-44

[47] J. Li, G. Brader, E.T. Palva, The WRKY70 transcription factor: a node of convergence for jasmonate-mediated and salicylate-mediated signals in plant defense, Plant Cell 16 (2004) 319-331.

[48] A. Méndez-Bravo, C. Calderón-Vázquez, E. Ibarra-Laclette, J. Raya-González, E. Ramírez-Chávez, J. Molina-Torres, A.A. Guevara-García, J. López-Bucio, L. Herrera-Estrella, Alkamides activate jasmonic acid biosynthesis and signaling pathways and confer resistance to Botrytis cinerea in Arabidopsis thaliana, PLoS One 6 (2011) e27251. 